\title{
Theoretical aspects of the integration of thermography and pyrometry methods
}

\author{
by Nataliya Hots, Tadeusz Piątkowski
}

"Lviv Politechnic" National University, Ukraine

Military University of Technology, Warszawa, Poland

Both thermovision and pyrometric measuring are widely used for the control of industrial process thermo modes, the technical service and diagnostics of equipment as well as the works on security and safety provision.

The objective of thermovision measuring is the visualization and research of an object thermo field on the basis of temperature gradient identification at defined temperature sensitivity. The main purpose of pyrometric measuring is the identification of temperature values in certain object points with certain accuracy.

Nowadays, it is expediently to solve the complex task based on both methods advantages' unification in many technological processes: identification of object thermo field point temperature values with the needed accuracy. For this purpose it is necessary to integrate the methods of thermography and pyrometry on the basis of usage of these methods' advantages as well as common disadvantages' correction.

Both thermovision and pyrometric measuring are tightly connected between themselves. When different tasks are available, they are based on common laws, have similar principles of measuring system construction, and moreover, they have common problems. Along with small values of measuring means, reaching $(0,1 \div 1,0) \%$ [1], and their high temperature sensitivity, up to $(0,5 \div 1,0){ }^{\circ} \mathrm{K}[1,2]$, the uncertainty of measurement results can make hundreds of degrees.

The low accuracy of temperature identification as a result of non-adequacy of the accepted mathematical model of a temperature measurement object due to eradiation and real physical phenomena belongs to shared disadvantages. The main factors causing the uncertainty of a measurement result are presented in the figure 1.

The mathematical model of the output signal of pyrometric transformer, which takes into account all the factors viewed above is shown by equation:

$$
\begin{aligned}
& U_{i}(T, \lambda, t)=U_{u}+U_{c o}+\frac{\pi k^{2} D_{0}^{2}}{16} \cdot k_{n} \cdot S_{\text {ін }} \cdot\left[\int_{\lambda_{1}}^{\lambda_{2}} \varepsilon(T, \lambda, \varphi, t) \cdot \tau_{\text {атиа }}(\lambda, t) \cdot \Psi(\lambda) \cdot b_{T}(T, \lambda, t) d \lambda+\right. \\
& \left.\left.+k_{k} \cdot \int_{\lambda_{1}}^{\lambda_{2}}[1-\varepsilon(T, \lambda, \varphi, t)] \cdot \varepsilon_{\text {ефф }}(T, \lambda, \alpha, t) \cdot \tau_{a}(\lambda, t) \cdot \Psi(\lambda) \cdot b_{\text {фо }}\left(T_{\phi}, \lambda, t\right) d \lambda\right]\right]
\end{aligned}
$$

$$
\text { according to: } \Phi_{\mathrm{i}}=\mathrm{f}\left(\varphi_{\mathrm{i}}\right) ; 0<\varepsilon(\mathrm{T}, \lambda, \varphi, \mathrm{t})<1 ;-90^{\circ} \leq \varphi \mathrm{l} \leq 90^{\circ} \text {, }
$$

where UO and Uc - output signals of the pyrometric transformer proportional to the additive error of measurement and the radiant flux of the intermediate medium; $k$-index of viewing(coefficient depending on the distance) ; D0 -diameter of the optical system; kn - coefficient of transformation Sir - integrated sensitivity of the infrared sensor; $\mathrm{Ri}$ - the coefficient, the value of which depends on the change of the angle of viewing; $\varphi$ - angle of radiation of the tested area of the surface; $\lambda 1-\lambda 2$ - spectral range of the infrared sensor; $\varepsilon(T, \lambda, t)-$ emissivity of the area of the surface; $\operatorname{Tc}(\lambda, t)$-transmittance of the intermediate medium; $\Psi(\lambda)$ - apparatus function of the means of measurements; bT 


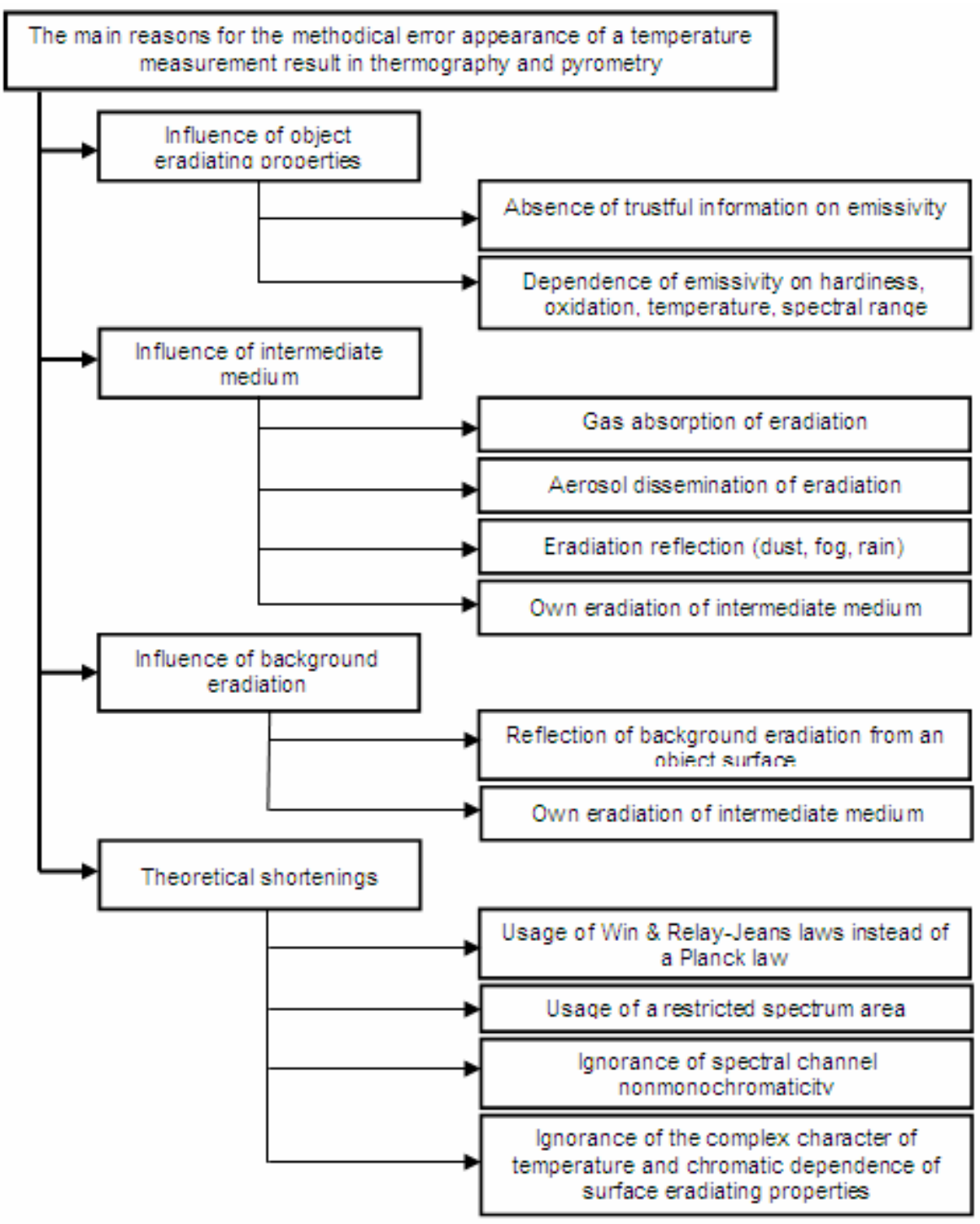

Fig. 1. The main reasons for the methodical error $y$ appearance of a measurement result in thermography and pyrometry

$(T, \lambda, t)$ - density of radiation flow of perfectly black body at the area temperature of the object measured; $\mathrm{k}_{\mathrm{k}}$ - angular coefficient of the background radiation; $\varepsilon \operatorname{epp}(\mathrm{T}, \lambda, \mathrm{t})$ - emissivity of the background radiation; bo $(T \phi, \lambda, t)$ - density of the radiation flow of the background radiation .

The factors causing the methodical error of measurement results and the ways of their reducing are analyzed. The influence of three main factors on the methodical error of measurement results is explored.

The influence of atmosphere on the temperature measurement resects is explored. The intervals of wave lengths which are supposed to be used for certain range temperature measurement are determined on the basis of the research of central values of atmosphere main component eradiation lengths (Table1). 
Table.1 The central meanings of wavelength of spectral absorption of radiation by the atmosphere

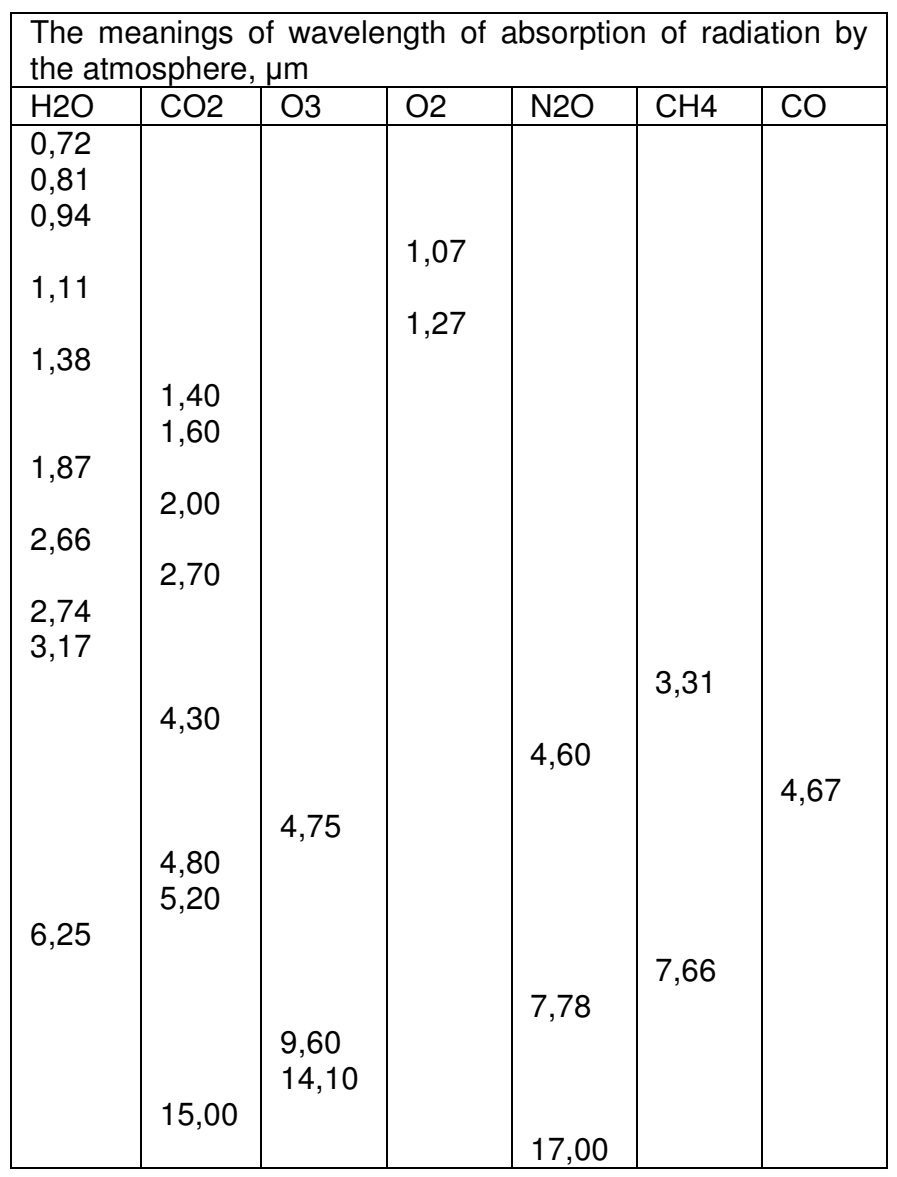

The absorption of radiation at the atmosphere (the length waves $0,7 \mu \mathrm{m} \div 17 \mu \mathrm{m}$ ) is explore and the central meanings of wavelength, where the eradiation reach more than $50 \%$ are defined. At the spectral ranges $3 \div 5 \mu \mathrm{m}$ and $8 \div 14 \mu \mathrm{m}$ many wavelength where absorption of radiation is $70-90 \%$ exist. That is why it is necessary to use the narrow spectral ranges througth the presented meanings of the wavelength. It reduces the methodical error of the measurement, caused by atmosphere absorption.

The exploration of dependence of a different materials' eradiation coefficient on temperature and wavelength is conducted (fig.2). The influence of difference of the eradiation properties of the real surface from blackbody on the methodical error of the measurement results is explored.

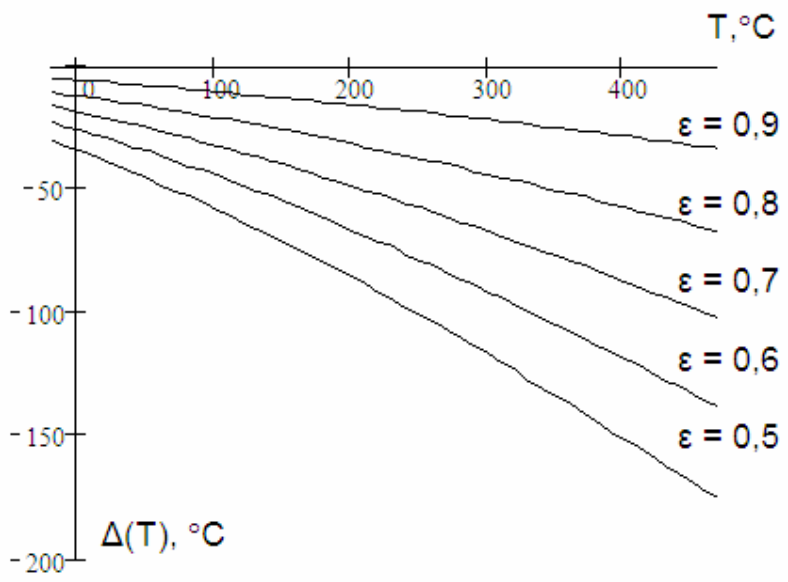

Fig 2. The methodical error of the temperature measurements caused by difference of the emissivity of the real surface and blackbody 
As a result of pyrometry methods' analysis conducted in (3), it is shown that multispectral pyrometry methods enable us to increase the accuracy of temperature measurement in certain conditions. Therefore they are supposed to be used in the thermography.

The influence of background eradiation of different intensity on the results of temperature measurement is explored. The conditions of the possible ignorance of background eradiation are defined. As a result of given experiments, measurement result methodical error caused by the influence of considered factors is estimated.

$$
\Delta(T),{ }^{\circ} \mathrm{C}
$$

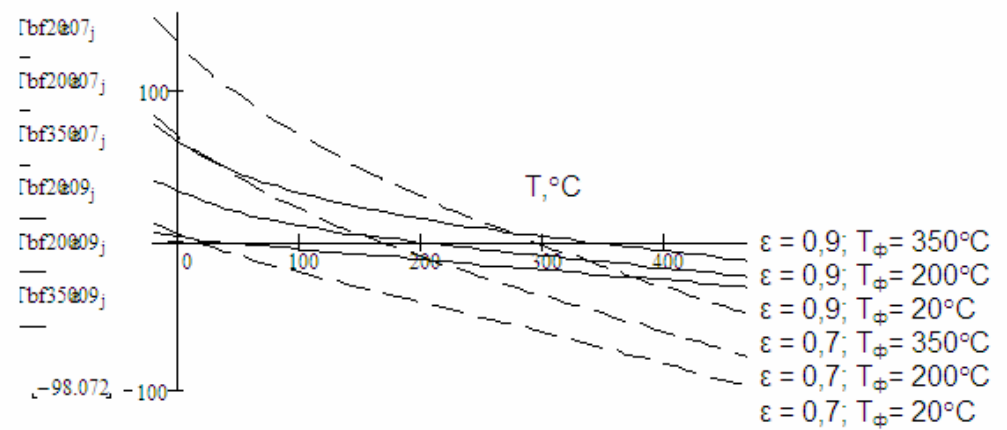

Fig.3 The metodical error of the temperature measurements caused by influence of background radiation

The influence of the reflective background radiation and its polymultiple reflectivity of the measurement results is explored. The methodical error of the temperature measurement caused by these factors is estimated.(fig.3 end fig.4).

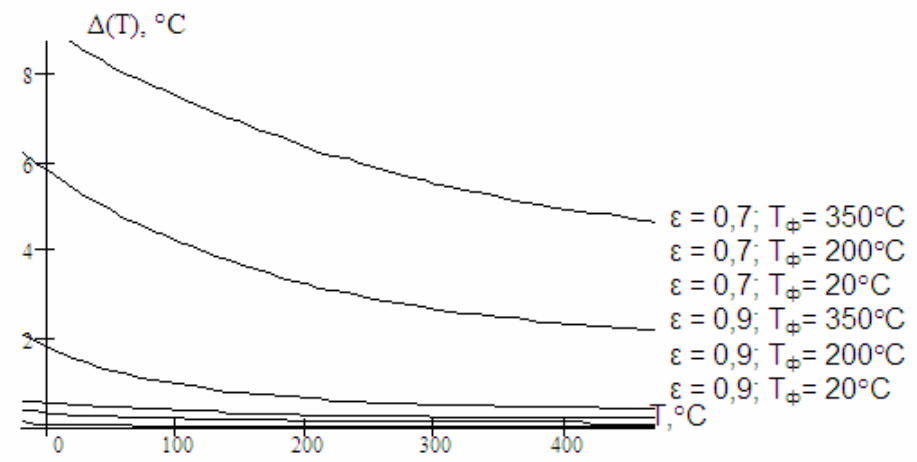

Fig.4 The methodical error of the temperature measurement caused by polymultiple reflectivity of background radiation

To reduce the methodical error from the influence of background radiation it is necessery to apply the following actions.

- To increase the emissivity of the measurement object;

- To equalize the background radiation temperature with temperature of the measurement object;

- To use a screen;

- To reduce the influence of background radiation choosing the spectral range where the influence is minimum.

- To use the differential methods of measurement.

Thus wider implementation of pyrometry precision methods in thermographical measuring enables us to decrease measurement result error and to widen their functional facilities.

\section{REFERENCES}

[1] Gots N. The Analysis Monochromatic Multichannal Pirometry. Abstract of $\mathrm{V}$ international conference PROBLEM OF INDUTRIAL HEAT ENGINEERING.-Kyiv, Ukraine.- 2007,- s.300-302.

[2] Гоц Н.Е. Сравнительная характеристика методов пирометрии / Научнщ-технический журнал «Приборы +Автоматика»,- Обнинск, Россия.- №7.- 2007, с.20-33. (По метериалам доклада на Третьей Всероссийской конференции по проблемам термометрии «Температура 2007» (Обнинск, Россия))

[3] Gilbert Gaussorgues. La Thermographie Infrarouge: Principes-Technologie-Applications. Paris.-LAVOISIER.1999,- 586p. 